\title{
Response of Sclerotinia Sclerotiorum to Long Exposure of Isothiocyanates by Transcriptomics Approach
}

\section{Pari Madloo}

University of Santiago de Compostela

Margarita Lema

University of Santiago de Compostela

Maria Elena Cartea

Misión Biológica de Galicia

Pilar Soengas ( $\square$ psoengas@mbg.csic.es )

Group of Genetics, Breeding and Biochemistry of Brassicas, Misión Biológica de 5 Galicia, Spanish Council for Scientific Research (MBG-CSIC), Pontevedra, Spain.

\section{Research Article}

Keywords: plant-pathogen interaction, glucosinolates, plant secondary metabolites, Brassica

Posted Date: January 18th, 2021

DOI: https://doi.org/10.21203/rs.3.rs-145054/v1

License: (c) (i) This work is licensed under a Creative Commons Attribution 4.0 International License. Read Full License 
1 Response of Sclerotinia sclerotiorum to long exposure of isothiocyanates by

2 transcriptomics approach

3

4 Pari Madloo ${ }^{1,2}$, Margarita Lema ${ }^{2}$, Maria Elena Cartea ${ }^{1}$, Pilar Soengas ${ }^{1 *}$

$5{ }^{1}$ Group of Genetics, Breeding and Biochemistry of Brassicas, Misión Biológica de

6 Galicia, Spanish Council for Scientific Research (MBG-CSIC), Pontevedra, Spain.

$7{ }^{2}$ Department of Functional Biology, Faculty of Biology, University of Santiago de 8 Compostela, Spain

9

10

11 *Corresponding author: psoengas@mbg.csic.es

12

13 Keywords: plant-pathogen interaction, glucosinolates, plant secondary metabolites, 14 Brassica.

15

16

17 


\section{Abstract}

20 White mold disease, caused by the necrotrophic fungus Sclerotinia sclerotiorum affects

21 Brassica crops. Brassica crops produce a broad array of compounds such as

22 glucosinolates that contribute to defense against pathogens. From their hydrolysis arise several products with antimicrobial activity called isothiocyanates (ITCs) which toxicity is structure-dependent. It is recognized that S. sclerotiorum is able to overcome the toxic effect of moderate concentrations of ITCs after a prolonged exposure to their action. Our objective was to identify the molecular mechanism underlying response of $S$. sclerotiorum to long exposure of two chemically diverse ITCs: the aliphatic allyl-ITC (AITC) and the indolic indol-3-carbinol (I3C). We found, with a transcriptomic approach, that the response is dependent of the type of ITC and to their initial target, involving cell membranes in the case of AITC or DNA in the case of I3C. Mechanisms of response include the reorganization of chromatin, mediated by histones chaperones hip4 and cia1, synthesis of ribosomes controlled by the pair kinase-phosphatase aps1ppn1, catabolism of proteins, synthesis of ergosterol and induction of efflux-pumpers and glutathione transferases. These mechanisms probably help S. sclerotiorum to grow and survive in an environment where ITCs are constantly produced by Brassica plants upon breakdown of glucosinolates. 
Sclerotinia sclerotiorum is a necrotrophic fungal pathogen that attacks at least 408 species of plants from 278 genera, belonging to 75 families (Wang et al., 2019). S. sclerotiorum produces the so-called Sclerotinia stem rot or white mold in Brassica crops with symptoms that include rotting of leaves, stems and pods, causing important economic losses, especially in oil crops (Zhang et al., 2015).

Brassica plants are able to produce a broad array of compounds that contribute to defense against $S$. sclerotiorum. This includes regulatory compounds such as jasmonic, salicylic acid and ethylene as well as defensive compounds such as glucosinolates (Zhang et al., 2015). These are secondary metabolites, present in the Brassicaeae family, derived from amino acids. Depending on the side chain of the aminoacid, glucosinolates are classified as: aliphatic (derived from methionine, alanine, valine, leucine, and isoleucine), aromatic (derived from phenylalanine or tyrosine) and indolic (derived from tryptophan) (Zukalova \& Vasak, 2002). Glucosinolates are hydrolyzed by myrosinases enzymes into several chemically diverse products. Hydrolysis occurs upon tissue breakdown produced by the attack of herbivores or necrotrophic pathogens (Calmes et al., 2015). These products showed antimicrobial effects in vitro against a broad range of fungal and bacterial plant pathogens (Sotelo et al., 2015, Calmes et al., 2015). Among hydrolytic products, isothiocyanates (ITCs) are the most toxic ones, even at low concentrations (Pastorczyk \& Bednarek, 2016).

Glucosinolates and their hydrolytic products have a role in the defense against $S$. sclerotiorum in planta (Madloo et al., 2019) and in vitro (Sotelo et al., 2015, Rahmanpour et al., 2009). The response of fungus seems to be dependent of the chemical structure of ITC (Madloo et al., 2019). However, to date, mechanisms through which ITCs exerts their toxicity and the ways fungus can overcome it are not 
65 completely understood. Generally speaking, ITCs can act directly by covalent 66 binding/modification of certain nucleophile-containing proteins referred as target or 67 indirectly by activating gene transcription (Mi et al., 2011). S. sclerotiorum can 68 overcome toxic effects driven by sub-lethal concentrations of ITCs after short exposure, 69 basically by inducing antioxidant defenses and efflux pumpers (Rahmanpour et al., 70 2009), similarly to other necrotrophic fungi, such as Alternaria brassicicola (Sellam et 71 al., 2007). This would allow the initial colonization of the plant by the fungus. 72 However, as the disease progress, more glucosinolates are hydrolyzed, and therefore $S$. 73 sclerotiorum is exposed during all the period of infection to the action of ITCs. Besides, 74 in a plant there are at the same time glucosinolates of different chemical classes 75 hydrolyzing to different ITCs which toxicity is structure-dependent (Li et al., 2013). In 76 this line, this study aimed to identify molecular mechanism underlying response of $S$. 77 sclerotiorum to long exposure of two chemically diverse ITCs. Identifying mechanisms 78 of response will allow progressing our understanding on the interaction of Brassica 79 plants with S. sclerotiorum.

80 
Phenotypical effect of ITC treatments on S. sclerotiorum

85

Mycelium of S. sclerotiorum spent more time to reach the end of Petri dish when it was treated with ITCs. This effect was more evident when samples were treated with I3C (Figure 1A). Growth rate reached its maximum in the first day under control conditions and in the second day in treated samples, then it decay progressively in control and AITC treated samples (Figure 1B). However, growth rate of fungus treated with I3C stabilized in the period of 3-6 days and then it decayed again. Once mycelium of AITC and control samples reached the end of petri dishes they start producing sclerotia in the border. However, sclerotia of I3C treated samples was produced also in a middle point of petri dishes (Figure 1E). $100 \mu \mathrm{M}$ AITC provoked permeabilization of membrane and it was half of that by $70 \%$ ethanol (Figure 1C). No permeabilization effect was observed for I3C (Figure 1D).

97

\section{Transcriptomic analysis}

An average of 14960380, 16415912 and 10776800 tag sequences were obtained from controls and treated samples with AITC and I3C, respectively. To have a general overview of the results, GO analysis with all the annotated transcripts was done for each comparison (ITC vs control and AITC vs I3C) with GO-Tool2. We give a description of the most important features found in the category of biological process (BP) (Supplementary Table S1). 
Analysis of the whole transcripts resulted in 212 significantly enriched GO terms ( $\mathrm{p}$ $\leq 0.05$ and $-1 \leq \log _{2}$ fold change $\geq 1$ ), from which 181 were induced and 31 repressed.

111 The most significant induced GO terms were related to mitosis and cell division (i.e., 112 GO:1901989, GO:1901992, GO:1903047, GO:0051301). Other categories were 113 related to organonitrogen compound metabolic process (GO:1901564) and small 114 molecule metabolic process (GO:0044281). Besides, there is a large set of significantly enriched terms associated with positive regulation of proteosomal 116 protein catabolic process (GO:1901800, GO:0032436). The first six most 117 significantly repressed GO terms are related to toxin biosynthetic pathways (GO:1901376; GO:1901378; GO:0045461; GO:0045460; GO:0009403, GO:0009404). Several terms were related to apoptosis and cell death (GO:0006915, GO:0012501, GO:0008219; GO:0016265). Other significant terms included processes related to mitochondrial transmembrane transport (GO:1990542, GO:0045041), mitochondrial

122 DNA repair (GO:0043504), and maturation of LSU-rRNA (GO:0000463, 123 GO:0000470).

I3C vs Control

127 GO analysis of I3C vs control rendered 197 significant GO terms, from which, 190 were repressed and 7 induced. Significantly induced GO terms were mostly related to synthesis of aminoacids, including serine, (GO:0009070, GO:0009092), threonine (GO:0009088) and sulfur amino acids (GO:0000097). Besides, ubiquitin-dependent 131 protein catabolic process via the multivesicular body sorting pathway (GO:0043162) 
and glyoxylate metabolic process (GO:0046487) were also significant. A large part of significant GO repressed terms were related to translation and to ribosome biogenesis and assembly (GO:0002181, GO:0042273, GO:0042255). Among the most significant GO down-regulated terms we could found several related to stress and stimulus response (GO:1901700, GO:0042221, GO:0006970, GO:0009408, GO:0010035). Other significantly repressed terms were implicated in small molecule metabolic process and nucleobase-containing small molecule metabolic process (GO:0044281, GO:0055086) and toxin biosynthetic process (GO:0009403).

\section{AITC vs I3C}

Expression of 296 GOs was higher in mycelium treated with AITC than in mycelium treated with I3C. Among them we can find terms related to cell division, such as: positive regulation of metaphase/anaphase transition of cell cycle (GO: 1902101), positive regulation of cell cycle phase transition (GO: 1901989) or actin polymerization or depolymerization (GO:0008154). Other terms are related to catabolic processes such as: positive regulation of proteosomal protein catabolic process (GO:1901800), positive regulation of proteolysis (GO:0045862). Translation (GO:0006412), ribonucleoprotein complex assembly (GO:0022618), ER-nucleus signaling pathway (GO:0006984), cellular response to sterol depletion (GO:0071501) and fungal-type cell wall biogenesis (GO:0009272) were also up-regulated by AITC. The expression of four GO terms was higher in the treatment with $\mathrm{I} 3 \mathrm{C}$ compared to AITC: mitochondrial DNA repair (GO:0043504), cellular response to light stimulus (GO:0071482), mitochondrial DNA metabolic process (GO:0032042) and response to light stimulus (GO:0009416). 
Differential expressed genes

159

There was higher number of DEGs in response to AITC than in response to I3C compared to control. Thirty genes were commonly induced by both treatments, and 98 were repressed (Figure 2A, B). In the comparison AITC vs I3C, 291 DEGs were induced in response to AITC and 427 were induced in response to I3C (figure $2 \mathrm{C}$ ). The relationships among DEGS from each comparison were deduced by using the web-tool STRING (v10.5). One hundred twenty-three DEGS from AITC vs Control comparison interact in one single network (Figure 3). Confirming results from GO analysis, DEGs interact in several clusters related to sterol and lipid synthesis, proteasome, translation, cell division and DNA replication (Figure 3, Supplementary Table S2). Twenty-one DEGs interact in two different networks in the comparison I3C vs Control (Figure 4, Supplementary Table S2). Part of the structure of network 1 (including DEGs mad2, klp9, mug190, ish1, hsp9 and SPAC32A11) is shared with the network of the comparison AITC vs Control. In both comparisons, mad2 and klp9 are induced and mug190, ish1, hsp9 and SPAC32A11 are repressed. Seventy-four DEGs are interacting in the comparison AITC vs I3C (Figure 5, Supplementary Table S2) in two different networks. In network 1 there is a cluster of structural proteins related to ribosome synthesis, which are higher induced by AITC than by I3C. All these structural proteins are related to protein aps1, which is repressed by I3C and induced by AITC. In this network a cluster of DEGs related to ergosterol synthesis (induced by AITC) and leucine synthesis (induced by I3C) also appear, interacting by protein SPAC22A12.16.1. In network 2 several DEGs: hip4, hhf1, mst1, ngg1, are induced by AITC, whereas DEGS rhp6, ubc7, rev1 and rhp14, are induced by 
182 I3C. Both branches are joined by DEG cia1, which is induced by I3C and repressed by 183 AITC.

DEGs in the three comparisons that were related to xenobiotic transport, transfer 185 of glutathione and toxin biosynthesis are shown in Table 1.

186 


\section{ITCs alter fungus growth and cell cycle}

190

191 Treatment with both ITCs produced a slower growth of mycelium compared to control

192 conditions. This effect was more evident in fungus treated with I3C than with AITC.

Besides, in I3C treated samples number of transcripts found was lower than in AITC samples or control, indicating that at the same concentrations, I3C is more toxic than AITC on S. sclerotiorum.

Both treatments provoked an anti-apoptotic response. Necrotrophic fungi reduce growth and blocks host-induced programmed cell death to maintain at least few hypha bodies to survive in early stages of infection. In the second phase of infection, surviving hypha will start growing to produce new mycelium that will invade plant tissues producing lesions in the plant (Goncalves et al., 2017). Several common transcripts in both treatments have anti-apoptotic function, of which only casA and crz1 were significantly repressed by both ITCs and ppk31 was significantly repressed by AITC. CasA is a metacaspase-1, which in other fungi (Aspergillus nidulans and A. fumigatus) is a positive regulator of endoplasmatic reticulum (ER) stress induced cell death (Tsiatsiani et al., 2011). Crz1 is a target of the calcineurim protein that is related to resistance to antifungal molecules and cations homeostasis in Saccharomyces cerevisiae (Karababa et al., 2006).

Ppk31 is involved in the induction of autophagy and in the regulation of lifespan. Ppk31 increases of chronological lifespan of the S. pombe DY8531 strain (Ellis et al., 2018). Lifespan can be modified by changing the number of mitotic divisions that a cell can undergo during its lifetime, or by modifying the time, a cell 
212 remains viable. In the first case, the term employed is replicative lifespan and 213 chronological lifespan in the second case (Ellis et al., 2018). Therefore, by inhibiting 214 ppk31, fungus treated by AITC may stop chronological lifespan to increase replicative 215 lifespan. Unlike AITC, there appeared to be no increase in expression of GOs associated with mitosis in mycelium treated with I3C. Chronological lifespan in S. cerevisiae is related to amino acid availability. The addition of isoleucine, valine, and leucine extended chronological lifespan in $S$. cerevisiae on media with limitation of nitrogen and carbon sources (Alvers et al., 2009). Accordingly, in the comparison AITC vs I3C, DEGs leu1 and leu2, key genes in the synthesis of leucine were induced by I3C.

222 Besides, this DEGS co-expressed with oac1. Oac1 exchanges isopropylmalate by oxalacetate from mitochondria to cytosol. Leucine synthesis is related to this transporter, since its precursor pyruvate can be obtained by oxaloacetate, once this is in the mitochondria (Marobbio et al., 2008). (Tsang et al., 2015) proved that oac1 is related to life span extension in S. cerevisiae through regulation of NAD+. In the GO analysis, glyoxylate cycle was up-regulated by I3C compared to control. When simple sugars are scarce, lower eukaryotes employ glyoxylate cycle to obtain energy from twocarbon compounds (Liberti et al., 2013). There is a link between glyoxylate cycle and chronological lifespan in $S$. cerevisiae. Icl1 is a key gene in glyoxylate cycle and it was induced by I3C. Deletion of this gene in S. cerevisiae under calorie restriction provokes 232 a decrease in chronological lifespan (Orlandi et al., 2013). Following (Orlandi et al., 2013), chronological lifespan is favored by glyoxylate/gluconeogenesis flux contrarily to the flux of ethanol and acetate via Krebs cycle and mitochondrial respiration. One of the responses of fungus treated with I3C may be to increase the chronological lifespan 
on mycelium cells. Therefore, it seems that fungus is following two different growth strategies to cope with the toxic effects of both ITCs.

Both ITC provoke a delay in growth of mycelium. However, once fungus treated with AITC start growing, the shape of the graph of growth on time is similar to that of control. Rate of growth decreases on time until fungus reaches its maximum size, coinciding with the end of Petri dish. Therefore, it seems that AITC is provoking a delay in the normal cell cycle of fungus. However, I3C treated samples shows a different growth pattern. Between days 3-6 samples the rate of growth stabilizes and then, on day 7 starts decaying again until reaching the end of Petri dish. Differential expression on genes related to cell cycle may underlie differences in growth patterns.

However, although fungus treated with both ITCs showed different growth pattern, there are several transcripts related to cell cycle that were commonly regulated in the comparisons AITC vs Control and I3C vs Control. DEGs mad2 and klp9 were induced by both ITCs. They are related to spindle dynamics during mitosis (Fu et al., 2009, Moyle et al., 2014). Ish1 and hsp9 are part of the mitogen-activated protein kinase (MAPK) pathway and both are down-regulated by both ITCs. The expression of ish1 increases after exposure of $S$. pombe to osmotic stress or glucose deprivation (Taricani et al., 2002). The function of hsp9 is related to regulation of cell cycle by increasing the length of $S$. pombe cells and decreasing their growth rate (Ahn et al., 2012).

Other common DEGS were regulated differentially by both treatments in the comparison AITC vs I3C. DEGS of network 2 are mostly related to modification of histones and DNA repair. Synthesis of histone H4 (DEG hhf1) is induced by AITC. Histone chaperones modulates nucleosomes assembly and therefore, the structure of chromatin (Amin et al., 2012). Hip4 and cia1 in S. pombe (homologous to hpc2 and asf1 of S. cerevisiae, respectively) are chaperones acting on histones H3 and H4. Hip4 is a 
component of the HIR complex. Deletion of hip4 provokes cycle delay (Anderson et al., 2010). This gene is necessary for accurate chromosome segregation. Cia1 is a repressor of the HIR-dependent histone genes. Mutants in cial causes double strand breaks in DNA, increase DNA sensitivity to nuclease, and subsequently activate the DNA damage checkpoint pathway. Consequently, lifespan is severely reduced (Tanae et al., 2012, Anderson et al., 2010). AITC induces hip4 and I3C induces cia1.

The function of histone chaperones can be regulated by post-transcriptional modifications, leading to a regulation of different processes involving DNA, such as transcription, reparation or condensation (Zofall \& Grewal, 2007). DNA replication is regulated by the acetylation of histones H3 and H4 (Sklenar \& Parthun, 2004). This function is carried on by DEGS ngg1 and mst1 (Ruan et al., 2015), when fungus is treated with AITC. In the other hand, ubiquitination of DNA is induced by I3C, by means of rhp6 and rhp14. Both DEGS, together with the DNA polymerase rev1 act in DNA repair (Meyer et al., 2019, Cui et al., 2018, Hohl et al., 2001), contributing to genome stability.

Therefore, there is a regulation of chromatin structure dependent on ITCs. In the case of AITC treated samples, this reorganization seems to be involved in mitosis and DNA replication, whereas in the case of $\mathrm{I} 3 \mathrm{C}$ it may be related to DNA repair. Then differences in growth performance between both samples can be due to DNA reparation, being DNA a possible direct or indirect target of I3C.

\section{ITCs alter metabolism of proteins and lipids} mitosis of AITC treated cells is induced, they need to produce new proteins. However, 
in the case of I3C cells, which growing rate is slower, and needs to repair its DNA, ribosome synthesis is a high-consuming energy process. Eukaryotic cells cease the production of ribosomes very rapidly upon unfavorable conditions in order to ensure survival (Drygin et al., 2010). In the network created with DEGS of the comparison AITC vs I3C, a big cluster of ribosome-associated proteins is induced by AITC. All these proteins are linked to asp1, which is induced by I3C.

The inositol pyrophosphates (PP-InsPs) play a central role in signaling and reprogramming cell metabolism through their phosphorylation/dephosphorylation. Asp1 is a inositol kinase, which participates in the synthesis of the diphosphate group of PPInsP5 and (PP)2-InsP4 (Wu et al., 2016). Wu et al., (2016) found that a large number of proteins isolated from S. cerevisae that interact with 5PP-InsP5 are related to ribosome biogenesis and maturation. Asp1 co-expresses with ppn1, which is an endopolyphosphatase and catalyzes the hydrolysis of polyphosphate chains. This last DEG was induced by AITC. Therefore, it seems that the system aps1-ppn1 is controlling the synthesis of ribosomes in fungus treated with ITCs.

Both treatments induced catabolism of proteins. AITC induced the ubiquitinproteasomal system, whereas I3C induced the multivesicular (MVB) body sorting pathway. The ubiquitin-proteasome system has an essential role in cell growth and differentiation, survival, stress tolerance and adaptation to environmental changes (Mi et al., 2011, Piper \& Katzmann, 2007). This mechanism is critical for cell survival (Huang et al., 2001). Formation of MVB takes place by invagination of the membrane of an endosome. MVB are degraded by fusing to lysosomes (Piper \& Katzmann, 2007). Both mechanisms serve to regulate the turnover of proteins and to degrade the damaged ones. Proteins to be degraded by both systems are joined to ubiquitin as a signal (Piper \& Katzmann, 2007). In the comparison AITC vs I3C, two ubiquitination-related DEGS 
were significantly expressed. Ubc11 was induced by AITC, whereas, Ubc7 was induced by $\mathrm{I} 3 \mathrm{C}$.

Catabolism of proteins can be associated to mitosis and also to damage caused by ITC treatments. Secretory proteins are primarily assembled and folded in the endoplasmic reticulum (ER). Increment in misfolded proteins inside ER lumen can be provoked by and increment in the protein load but also it can respond to oxidative stress and to modifications in lipids and calcium (Volmer \& Ron, 2015). Lipid perturbation seems to happen in cells treated with AITC because this treatment diminished permeabilization of S. sclerotiorum cells, whereas I3C had no effect on it. Sterol biosynthetic process was induced in fungus treated with AITC in comparison to I3C. Sterols play an important structural role to regulate membrane fluidity and permeability (Parks \& Casey, 1995). Besides, DEG $w A$ was induced by AITC while repressed by I3C. This gene is related to the synthesis of melanin in fungi, which gives strength and rigidity to the cell wall and consequently it is related to cell permeability and turgor (Cordero \& Casadevall, 2017). In the comparison AITC vs I3C DEG plh1 was induced by I3C. This DEG codifies for a phospholipid: diacylglycerol acyltransferase, related to the synthesis of triacylglycerols (Feng et al., 2019). Fungus treated with AITC probably modify the composition of cellular membranes by increasing the level of sterols in comparison with control and $\mathrm{I} 3 \mathrm{C}$ treatments and decreasing tryacylglycerols in order to al., 2007, Somani et al., 2019, Bastos et al., 2019). 
338 Exposure to AITCs causes oxidative damage in fungi (Rahmanpour et al., 2009, Calmes 339 et al., 2015, Sellam et al., 2007). One of the possible explanations is that conjugation of 340 glutathione with ITCs may cause a drop in the free glutathione and the inhibition of 341 glutathione reductase. Conjugation of glutathione to ITCs is catalyzed by glutathione-Stransferases. As a mechanism of detoxification, glutathione transferases were induced by S. sclerotiorum (Rahmanpour et al., 2009), and A. brassicicola (Sellam et al., 2006, Calmes et al., 2015) after exposure to several ITCs. In line with this, several glutathione transferases were induced by both treatments, coincidently, SS1G_10295 was also induced after treatment of S. sclerotiorum with AITC (Rahmanpour et al., 2009). stress. Hog1 was induced after exposure of A. brassicicola to AITC (Calmes et al., 2015, Joubert et al., 2011). Hog1 and ras1 controlled the response of osmotic stress in Cryptococus neoformans exposed to amphotericin B (Sun et al., 2013). Signal transduction pathways involving MAP kinases are one of the major mechanisms that fungus employ to sense and response to environmental changes. However, in this work, sty1 (homologous to hog1) and ras1 were repressed after AITC treatment, probably because at the time point when samples were taken, osmotic and/or oxidative stress caused by AITC were reduced after several days of adaptation.

Induction of MFS and ABC transporters is a common mechanism of defense in order to detoxify fungi cells (Somani et al., 2019, Vela-Corcia et al., 2019, Sellam et al., 2007). In this case, several MFS and one $A B C$ transporters were induced by ITC 
specificity of substrate. MFS transporter SS1G_12101 was also induced in $S$. sclerotiorum after invasion of B. napus plants (Seifbarghi et al., 2017). In GO analysis, toxin biosynthetic process was down-regulated by both ITCs. This GO term include genes similar with sterigmatocystin coding genes. Sterigmatocystin is a polyketide compound that is produced by several Aspergillus species and is a precursor of aflatoxin. Repression of toxin related genes by fungi in the presence of fungicides has previously been described (Somani et al., 2019) and it may respond to a need of derive energy to primer metabolism.

\section{Conclusions}

Moderate concentrations of ITCs inhibit growth of S. sclerotiorum in vitro. However, after long-exposure fungus is able to overcome their toxic effects and growth at the same level as non-treated fungus. We give and overview of the mechanisms of adaptation of S. sclerotiorum to two chemically diverse ITCs, the aliphatic AITC and the indolic I3C. We show that mechanisms of adaptation are dependent of the type of ITC and to their initial target, involving cell membranes in the case of AITC or DNA in the case of I3C. Mechanisms of adaptation include the reorganization of chromatin, mediated by histones chaperones hip4 and cia1, synthesis of ribosomes controlled by the pair kinase-phosphatase aps1-ppn1, catabolism of proteins, synthesis of ergosterol and induction of efflux-pumpers and glutathione transferases. These mechanisms probably help $S$. sclerotiorum to grow and survive in an environment where ITCs are being produced by Brassica plants upon breakdown of glucosinolates. 


\section{Fungus growth and treatment with ITCs}

We collected the $S$. sclerotiorum isolate MBG-Ss2 employed in the experiments on $B$. napus in Spain and it is currently maintained at MBG-CSIC. In order to provide fresh colonies for experiments, surface sterilized sclerotia were cultivated on potato dextrose agar (PDA, Difco) plates and incubated at $24{ }^{\circ} \mathrm{C}$ in the dark for 4 days. Fresh colonies were obtained through routinely growth of mycelium-agar plugs from the margin of the fungus colony on PDA and incubated at $24^{\circ} \mathrm{C}$ for $72 \mathrm{~h}$. ITCs were purchased from SIGMA-ALDRICH including allyl-ITC (AITC) (36682) and indole-3-carbinol (I3C) (I7256-5G). Minimal inhibitory concentration which resulted in complete growth inhibition of fungus was tested for each ITC. The growth of fungus in 2x YPG media was monitored spectrophotometrically for 4 days at $25^{\circ} \mathrm{C}$ with the addition of each ITC in concentrations ranging from 0 to $250 \mu \mathrm{M}$. Then a sub-lethal concentration of $100 \mu \mathrm{M}$ for both compounds was selected to use in transcriptomic analysis.

400

Fungus was grown in PDA medium supplemented with each ITC, which was added to the medium before solidification to a concentration of $100 \mu \mathrm{M}$. Petri dishes were coated with one layer of cellophane membrane (Bio-Rad Catalog No. 165-0963). Then, agar plugs of $2.5 \mathrm{~mm}$ diameter containing fresh fungus colonies were transferred to petri dishes and incubated at $24^{\circ} \mathrm{C}$ in darkness. After 5 days mycelium mass was harvested from cellophane membranes and homogenized in liquid nitrogen. The resultant material was used immediately for isolation of total RNAs. Two replicates per treatment and control samples were carried out. 
In other experiment, diameter of fungal mycelium growing radially from the center was measured during 8 days, until reaching the end of petri dishes. Growth rate was computed between time points as follows: (growth $t_{i+1}$-growth $t_{i}$ )/ growth $t_{i+1}$, varying $i$ from 1 to 7. Experimental conditions were the same as in RNA experiment. Three replicates were done for each treatment.

\section{RNA isolation, library preparation and sequencing}

RNA was extracted with Spectrum ${ }^{\mathrm{TM}}$ Plant Total RNA Kit (Sigma MO, USA) according to the manufacturer's protocol. To remove any traces of genomic DNA, the RNA was treated with DNase by following the manufacturer's instructions. Massive Analysis of 3'-cDNA Ends (MACE) libraries were generated using GenXPro's MACE kit (GenXPro GmbH, Frankfurt, Germany) as described by Zawada et al. (2014). Briefly, cDNA from $2 \mu \mathrm{g}$ of total RNA was randomly fragmented and biotinylated 3' ends were captured after binding to a streptavidin matrix. A library ready for highthroughput sequencing was prepared using TrueQuant adapters included in the kit. The library consisted of 50-700 bp-long fragments derived from the 3 '-end of the cDNAs. The 5'-ends of the libraries were sequenced using an Illumina Hiseq 2000 version 4 chemistry (Illumina, Inc., San Diego, CA, USA).

NextSeq -derived sequence reads were processed with GenXPro's online inhouse MACE analysis pipeline. Briefly, libraries were sorted according to their respective index and all duplicate reads detected by the TrueQuant technology were removed from the raw datasets. Additionally, quality filtering (by software Cutadapt (https:// github.com/marcelm/cutadapt/)), and poly(A)-tails were clipped by an in-house python-script. The reads were aligned to reference sequences using "bowtie2". On this 
genome, as the functional annotation was poor, a "cluster_bed" step was performed.

Reads that map successfully to the genomic regions with no annotation (feature) were

clustered. The clusters (consensus sequences) were annotated via BLASTX to the

Uniprot database (Swiss-Prot and TrEMBL). Reads that did not match the genome were taken into a de novo assembly step and annotated via BLASTX to the Uniprot database (Swiss-Prot and TrEMBL). The assembly was done with Trinity RNA-Seq (https://github.com/trinityrnaseq/trinityrnaseq/wiki). For the functional annotation Uniprot (http://www.uniprot.org/) and NCBI fungal sequences were employed

\section{Bioinformatic analysis}

To have a general overview of the results, GO analysis with all the annotated transcripts was done for each comparison (ITC vs control and AITC vs I3C) with GO-Tool2. The likelihood of a specific GO term was calculated using the Fisher's exact test. change (FC) $\geq 1$ were considered to be differentially expressed genes (DEGs) between treatments. The web-tool STRING (v. 10.5) was used in order to study the interconnections between DEGs. This resource, in addition to well-supported protein451 protein interactions experimentally observed, includes indirect and predicted interactions on top (Szklarczyk et al., 2017). However, there is little information about interactions among proteins in S. sclerotinia genome. Relationships among proteins were deduced employing the genome of Schizosaccharomyces pombe as reference, since $10 \%$ of annotations were found in this genome and there is much more experimental evidence of interactions among proteins than in S. sclerotiorum. 
457 Determination of membrane permeabilizing potential in $S$. sclerotiorum treated

458

459

460

461

462

463

464

465

466

467

468

469

470

471

472 with ITCs

The SYTOX ${ }^{\circledR}$-Green uptake assay was carried out following Theis et al. (2003). When plasma membrane integrity is compromised, influx of the dye and subsequent binding to DNA causes a large increase in fluorescence. In brief, $10 \mu 1$ of fungus mycelium suspension (OD 0.37) was added to $100 \mu \mathrm{l}$ of $2 \times \mathrm{YPG}$ liquid medium ( $\mathrm{pH} 4.5$ ) and incubated for $48 \mathrm{~h}$ at $27{ }^{\circ} \mathrm{C}$. Then, AITC or I3C was added to reach the final concentrations of 50, 75 and $100 \mu \mathrm{M}$. SYTOX-Green was added to a concentration of $0.2 \mu \mathrm{M}$. Samples showing $100 \%$ permeabilization were prepared by degrading cells with $70 \%$ ethanol. Fluorescence reads were carried out over a time course of $t=0$ to $t=$ 210 min using a Promega fluorescence measurement system (GloMax) at an excitation wavelength of $480 \mathrm{~nm}$ and an emission wavelength of $530 \mathrm{~nm}$. Fluorescence values were corrected by absorbance of samples measured at $600 \mathrm{~nm}$. Measurements were carried out in triplicate. 
Ahn, J., Won, M., Choi, J. H., Kyun, M. L., Cho, H. S., Park, H. M., et al. (2012) Small heat-shock protein Hsp9 has dual functions in stress adaptation and stressinduced G2-M checkpoint regulation via $\mathrm{Cdc} 25$ inactivation in Schizosaccharomyces pombe. Biochemical and Biophysical Research Communications, 417, 613-618.

Alvers, A. L., Fishwick, L. K., Wood, M. S., Hu, D., Chung, H. S., Dunn, W. A., et al. (2009) Autophagy and amino acid homeostasis are required for chronological longevity in Saccharomyces cerevisiae. Aging Cell, 8, 353-369.

Amin, A. D., Vishnoi, N. and Prochasson, P. (2012) A global requirement for the HIR complex in the assembly of chromatin. Biochim. Biophys. Acta-Gene Regul. Mech., 1819, 264-276.

Anderson, H. E., Kagansky, A., Wardle, J., Rappsilber, J., Allshire, R. C. and Whitehall, S. K. (2010) Silencing mediated by the Schizosaccharomyces pombe HIRA complex is dependent upon the Hpc2-like protein, Hip4. PLoS One, 5.

Bastos, R. W., Freitas, G. J. C., Oliveira, H., Oliveira, L. V. N., Gouveia-Eufrasio, L., Santos, A. P. N., et al. (2019) From the environment to the host: How non-azole agrochemical exposure affects the antifungal susceptibility and virulence of Cryptococcus gattii. Sci. Total Environ., 681, 516-523.

Calmes, B., N'Guyen, G., Dumur, J., Brisach, C. A., Campion, C., Iacomi, B., et al. (2015) Glucosinolate-derived isothiocyanates impact mitochondrial function in fungal cells and elicit an oxidative stress response necessary for growth recovery. Frontiers in Plant Science, 6. 
Cordero, R. J. B. and Casadevall, A. (2017) Functions of fungal melanin beyond virulence. Fungal Biology Reviews, 31, 99-112.

Cui, G. F., Botuyan, M. V. and Mer, G. (2018) Structural basis for the interaction of mutasome assembly factor REV1 with ubiquitin. J. Mol. Biol., 430, 2042-2050.

Drygin, D., Rice, W. G. and Grummt, I. (2010) The RNA polymerase I transcription machinery: An emerging target for the treatment of cancer. Annu. Rev. Pharmacol. Toxicol., 50, 131-156.

Ellis, D. A., Mustonen, V., Rodriguez-Lopez, M., Rallis, C., Malecki, M., Jeffares, D. C., et al. (2018) Uncovering natural longevity alleles from intercrossed pools of aging fission yeast cells. Genetics, 210, 733-744.

Feng, Y. B., Zhang, Y. X., Ding, W., Wu, P. C., Cao, X. P. and Xue, S. (2019) Expanding of phospholipid:diacylglycerol acyltransferase (PDAT) from Saccharomyces cerevisiae as multifunctional biocatalyst with broad acyl donor/acceptor selectivity. Appl. Biochem. Biotechnol., 188, 824-835.

Fu, C. H., Ward, J. J., Loiodice, I., Velve-Casquillas, G., Nedelec, F. J. and Tran, P. T. (2009) Phospho-regulated interaction between kinesin-6 Klp9p and microtubule bundler Ase1p promotes spindle elongation. Dev. Cell, 17, 257-267.

Gautam, P., Shankar, J., Madan, T., Sirdeshmukh, R., Sundaram, C. S., Gade, W. N., et al. (2008) Proteomic and transcriptomic analysis of Aspergillus fumigatus on exposure to amphotericin B. Antimicrob. Agents Chemother., 52, 4220-4227.

Goncalves, A. P., Heller, J., Daskalov, A., Videira, A. and Glass, N. L. (2017) Regulated forms of cell death in fungi. Front. Microbiol., 8.

Hohl, M., Christensen, O., Kunz, C., Naegeli, H. and Fleck, O. (2001) Binding and repair of mismatched DNA mediated by Rhp14, the fission yeast homologue of human XPA. J. Biol. Chem., 276, 30766-30772. 
Huang, Y. H., Shin, N. H., Sun, Y. and Wang, K. K. W. (2001) Molecular cloning and characterization of a novel caspase-3 variant that attenuates apoptosis induced by proteasome inhibition. Biochemical and Biophysical Research Communications, 283, 762-769.

Joubert, A., Bataille-Simoneau, N., Campion, C., Guillemette, T., Hudhomme, P., Iacomi-Vasilescu, B., et al. (2011) Cell wall integrity and high osmolarity glycerol pathways are required for adaptation of Alternaria brassicicola to cell wall stress caused by brassicaceous indolic phytoalexins. Cell Microbiol., 13, 62-80.

Karababa, M., Valentino, E., Pardini, G., Coste, A. T., Bille, J. and Sanglard, D. (2006) CRZ1, a target of the calcineurin pathway in Candida albicans. Mol. Microbiol., 59, 1429-1451.

Li, D. G., Shu, Y. A., Li, P. L., Zhang, W. B., Ni, H. W. and Cao, Y. S. (2013) Synthesis and structure-activity relationships of aliphatic isothiocyanate analogs as antibiotic agents. Medicinal Chemistry Research, 22, 3119-3125.

Liberti, D., Rollins, J. A. and Dobinson, K. F. (2013) Peroxysomal carnitine acetyl transferase influences host colonization capacity in Sclerotinia sclerotiorum. Mol. Plant-Microbe Interact., 26, 768-780.

Madloo, P., Lema, M., Francisco, M. and Soengas, P. (2019) Role of major glucosinolates in the defense of kale against Sclerotinia sclerotiorum and Xanthomonas campestris pv. campestris. Phytopathology, 109, 1246-1256.

Marobbio, C. M. T., Giannuzzi, G., Paradies, E., Pierri, C. L. and Palmieri, F. (2008) alpha-isopropylmalate, a leucine biosynthesis intermediate in yeast, is transported by the mitochondrial oxalacetate carrier. J. Biol. Chem., 283, 2844528453. 
Meyer, D., Fu, B. X. H., Chavez, M., Loeillet, S., Cerqueira, P. G., Nicolas, A., et al. (2019) Cooperation between non-essential DNA polymerases contributes to genome stability in Saccharomyces cerevisiae. DNA Repair, 76, 40-49.

Mi, L. X., Di Pasqua, A. J. and Chung, F. L. (2011) Proteins as binding targets of isothiocyanates in cancer prevention. Carcinogenesis, 32, 1405-1413.

Moyle, M. W., Kim, T., Hattersley, N., Espeut, J., Cheerambathur, D. K., Oegema, K., et al. (2014) A Bub1-Mad1 interaction targets the Mad1-Mad2 complex to unattached kinetochores to initiate the spindle checkpoint. J. Cell Biol., 204, 647-657.

Orlandi, I., Ronzulli, R., Casatta, N. and Vai, M. (2013) Ethanol and acetate acting as carbon/energy sources negatively affect yeast chronological aging. Oxidative Med. Cell. Longev.

Parks, L. W. and Casey, W. M. (1995) Physiological implications of sterol biosynthesis in yeast. Annu. Rev. Microbiol., 49, 95-116.

Pastorczyk, M. and Bednarek, P. (2016) The function of glucosinolates and related metabolites in plant innate immunity. In: Glucosinolates. (Kopriva, S., ed.). pp. 171-198.

Piper, R. C. and Katzmann, D. J. (2007) Biogenesis and function of multivesicular bodies. In: Annual Review of Cell and Developmental Biology. Palo Alto: Annual Reviews, pp. 519-547.

Rahmanpour, S., Backhouse, D. and Nonhebel, H. M. (2009) Induced tolerance of Sclerotinia sclerotiorum to isothiocyanates and toxic volatiles from Brassica species. Plant Pathol., 58, 479-486. 
Ruan, K., Yamamoto, T. G., Asakawa, H., Chikashige, Y., Kimura, H., Masukata, H., et al. (2015) Histone H4 acetylation required for chromatin decompaction during DNA replication. Sci Rep, 5.

Seifbarghi, S., Borhan, M. H., Wei, Y., Coutu, C., Robinson, S. J. and Hegedus, D. D. (2017) Changes in the Sclerotinia sclerotiorum transcriptome during infection of Brassica napus. BMC Genomics, 18.

Sellam, A., Dongo, A., Guillemette, T., Hudhomme, P. and Simoneau, P. (2007) Transcriptional responses to exposure to the brassicaceous defence metabolites camalexin and allyl-isothiocyanate in the necrotrophic fungus Alternaria brassicicola. Mol. Plant Pathol., 8, 195-208.

Sellam, A., Poupard, P. and Simoneau, P. (2006) Molecular cloning of AbGst1 encoding a glutathione transferase differentially expressed during exposure of Alternaria brassicicola to isothiocyanates. Fems Microbiology Letters, 258, 241-249.

Sklenar, A. R. and Parthun, M. R. (2004) Characterization of yeast histone H3-specific type B histone acetyltransferases identifies an ADA2-independent Gcn5p activity. BMC biochemistry, 5, 11.

Somani, D., Adhav, R., Prashant, R. and Kadoo, N. Y. (2019) Transcriptomics analysis of propiconazole-treated Cochliobolus sativus reveals new putative azole targets in the plant pathogen. Funct. Integr. Genomics, 19, 453-465.

Sotelo, T., Lema, M., Soengas, P., Cartea, M. E. and Velasco, P. (2015) In vitro activity of glucosinolates and their degradation products against Brassica-pathogenic bacteria and fungi. Applied and Environmental Microbiology, 81, 432-440.

Sun, N., Fonzi, W., Chen, H., She, X. D., Zhang, L. L., Zhang, L. X., et al. (2013) Azole susceptibility and transcriptome profiling in Candida albicans 

Chemother., 57, 532-542.

597

598

599

600

601

602

603

604

605

606

607

608

609

610

611

612

613

614

615

616

617

618

619

Szklarczyk, D., Morris, J. H., Cook, H., Kuhn, M., Wyder, S., Simonovic, M., et al. (2017) The STRING database in 2017: quality-controlled protein-protein association networks, made broadly accessible. Nucleic Acids Research, 45, D362-D368.

Tanae, K., Horiuchi, T., Matsuo, Y., Katayama, S. and Kawamukai, M. (2012) Histone chaperone Asf1 plays an essential role in maintaining genomic stability in fission yeast. PLoS One, 7.

Taricani, L., Tejada, M. L. and Young, P. G. (2002) The fission yeast ES2 homologue, Bis1, interacts with the Ish1 stress-responsive nuclear envelope protein. J. Biol. Chem., 277, 10562-10572.

Theis, T., Wedde, M., Meyer, V. and Stahl, U. (2003) The antifungal protein from Aspergillus giganteus causes membrane permeabilization. Antimicrob. Agents Chemother., 47, 588-593.

Tsang, F., James, C., Kato, M., Myers, V., Ilyas, I., Tsang, M., et al. (2015) Reduced Ssy1-Ptr3-Ssy5 (SPS) signaling extends replicative life span by enhancing $\mathrm{NAD}(+)$ homeostasis in Saccharomyces cerevisiae. J. Biol. Chem., 290, 1275312764.

Tsiatsiani, L., Van Breusegem, F., Gallois, P., Zavialov, A., Lam, E. and Bozhkov, P. V. (2011) Metacaspases. Cell Death Differ., 18, 1279-1288.

Vela-Corcia, D., Srivastava, D. A., Dafa-Berger, A., Rotem, N., Barda, O. and Levy, M. (2019) MFS transporter from Botrytis cinerea provides tolerance to glucosinolate-breakdown products and is required for pathogenicity. Nat. Commun., 10. 
Volmer, R. and Ron, D. (2015) Lipid-dependent regulation of the unfolded protein response. Curr. Opin. Cell Biol., 33, 67-73.

Wang, Z., Ma, L. Y., Cao, J., Li, Y. L., Ding, L. N., Zhu, K. M., et al. (2019) Recent advances in mechanisms of plant defense to Sclerotinia sclerotiorum. Frontiers in Plant Science, $\mathbf{1 0 .}$

Wu, M. X., Chong, L. S., Perlman, D. H., Resnick, A. C. and Fiedler, D. (2016) Inositol polyphosphates intersect with signaling and metabolic networks via two distinct mechanisms. Proceedings of the National Academy of Sciences of the United States of America, 113, E6757-E6765.

Zawada, A. M., Rogacev, K. S., Mueller, S., Rotter, B., Winter, P., Fliser, D., et al. (2014) Massive analysis of cDNA ends (MACE) and miRNA expression profiling identifies proatherogenic pathways in chronic kidney disease. Epigenetics, 9, 161-172.

Zhang, Y. Y., Huai, D. X., Yang, Q. Y., Cheng, Y., Ma, M., Kliebenstein, D. J., et al. (2015) Overexpression of three glucosinolate biosynthesis genes in Brassica napus identifies enhanced resistance to Sclerotinia sclerotiorum and Botrytis cinerea. PLoS One, 10.

Zofall, M. and Grewal, S. I. S. (2007) HULC, a histone H2B ubiquitinating complex, modulates heterochromatin independent of histone methylation in fission yeast. J. Biol. Chem., 282, 14065-14072.

Zukalova, H. and Vasak, J. (2002) The role and effects of glucosinolates of Brassica species - a review. Rostl. Vyroba, 48, 175-180. 
644 This research was supported by projects AGL2015-66256-C2-R and RTI2018-096591-

645 B-I00, trough the Spanish Ministry of Science and the European Regional Development 646 Fund (ERDF). Pari Madloo acknowledges a PhD student fellowship from Erasmus 647 Mundus Action 2 Program.

648 Author contributions

649 PM did the experimental work. ML and PS designed the experiments. PM, ML, MEC 650 and PS contributed to the discussion of the results. PM and PS wrote the manuscript.

651 Data Availability Statement

652 The data that support the findings of this study are openly available in GeneExpression 653 Omnibus repository at https://www.ncbi.nlm.nih.gov/geo/, reference number 654 GSE144344.

655

656

657 Figure legends

658

659 Figure 1. Radial growth A) and growth rate B) of Sclerotinia sclerotiorum in PDA 660 medium with $100 \mu \mathrm{M}$ of allyl-isothiocyanate (AITC) or indol-3-carbinol (I3C) 661 measured during eight consecutive days. Cell permeability assay of Sclerotinia 662 sclerotiorum treated with AITC C) and I3C D) 40 minutes after the addition of 663 SYTOX $^{\circledR}$-Green. Letters in the top of the bars indicate separation of means by least 
664

665

666

667

668

669

670

671

672

673

674

675

676

677

678

679

680

681

682

683

684

685

686

687

688

significant differences at $\mathrm{p} \leq 0.05$. Production of sclerotia of Sclerotinia sclerotiorum grown in PDA medium under three different treatments (from left to the right): Control, AITC and I3C.

Figure 2. Venn graph showing overlapping A) up-differentially expressed genes (DEGs) and B) down-DEGs between the comparisons AITC vs Control and I3C vs Control. C) Number of DEGs up-regulated by AITC or I3C in the comparison AITC vs I3C. Identification of DEGs was performed based on a false discovery rate (FDR) $\leq 0.05$ and $-1 \leq \log _{2}$ fold change $(\mathrm{FC}) \geq 1 \log _{2}$

Figure 3. Protein association network built with all DEGs of the comparison AITC vs Control. Line thickness indicates the strength of data support, which is based on experimental results and co-expression analysis in Schizosaccharomyces pombe. Blue and yellow nodes indicate up or down-regulation of DEGs, respectively. Name of DEGs cited in the text are written in green. The name of DEGS shared with the comparison I3C vs Control are shown in red. Description of DEGs is given in Supplementary Table $\mathrm{S} 2$.

Figure 4. Protein association network built with all DEGs of the comparison I3C vs Control. Line thickness indicates the strength of data support, which is based on experimental results and co-expression analysis in Schizosaccharomyces pombe. Blue and yellow nodes indicate up or down-regulation of DEGs, respectively. Name of DEGs cited in the text are written in green. The name of DEGS shared with the comparison I3C vs Control are shown in red. Description of DEGs is included Supplementary Table $\mathrm{S} 2$. 
690 Figure 5. Protein association network built with all DEGs of the comparison AITC vs 691 I3C. Line thickness indicates the strength of data support, which is based on 692 experimental results and co-expression analysis in Schizosaccharomyces pombe. Blue 693 nodes indicate up-regulated DEGs by AITC and yellow nodes indicate up-regulated 694 DEGs by I3C, respectively. Description of DEGs is included in Supplementary Table 695 S2.

696

697 Supplementary information legends

698

699 Supplementary Table S1: GO terms of Biological Processes with $\mathrm{p}$ value lower than $700 \quad 0.05$ and $\log 2>1$ in the comparisons AITC vs control, I3C vs Control and AITC vs I3C.

701

702 Supplementary Table S2: List of DEGs which show protein-protein interaction forming 703 networks in the comparisons AITC vs Control I3C vs Control and AITC vs I3C. 
Table 1. List of differentially expressed genes (DEGs) related to transport of genotoxics, transfer of glutathione and toxin biosynthesis. Annotations of DEGs were performed via BLASTX to the Uniprot database (Swiss-Prot and TrEMBL) and TrinityRNA-Seq. The false discovery rate (FDR) of each DEG and its $\log _{2}$ fold change are shown for each one of the comparisons where they are significant.

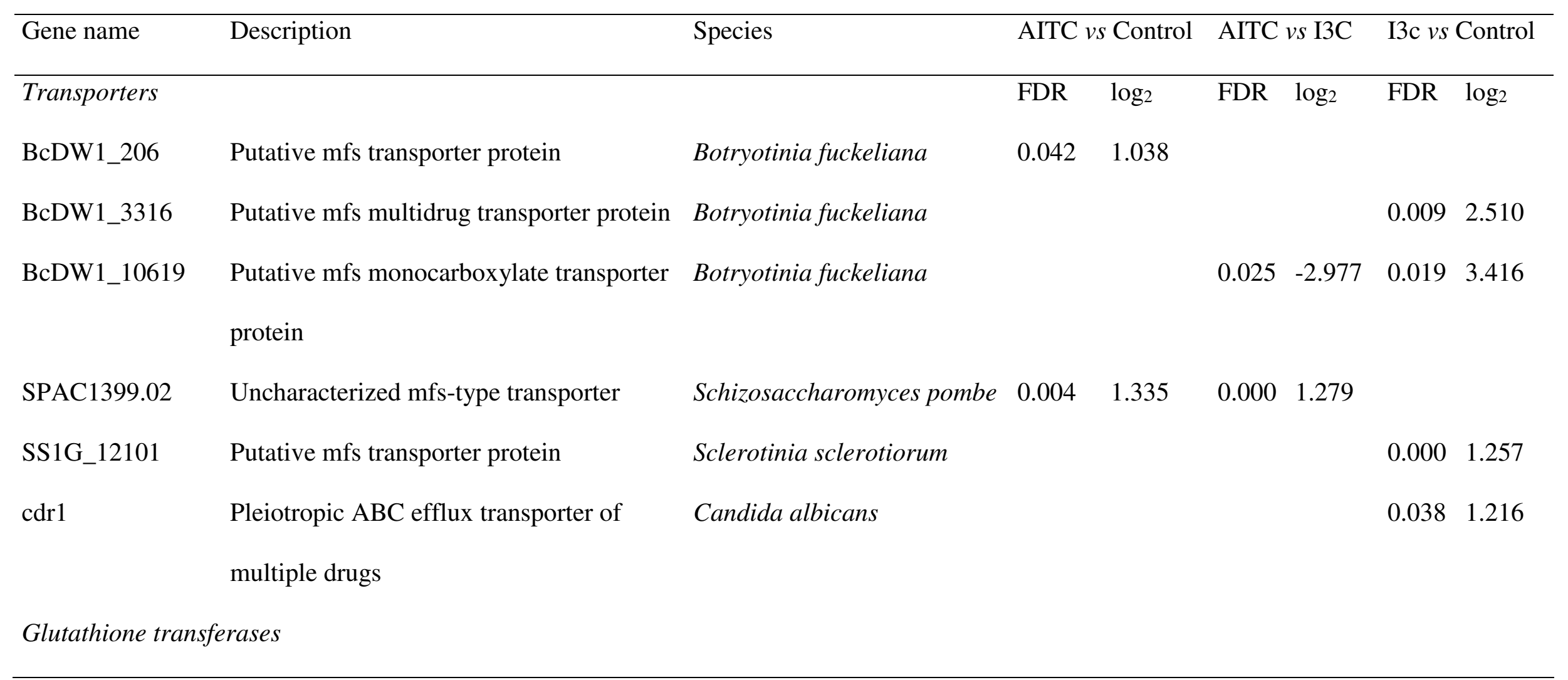




\begin{tabular}{|c|c|c|c|c|c|c|c|c|}
\hline SS1G_10295 & Putative glutathione S-transferase & Sclerotinia sclerotiorum & 0.001 & 2.466 & 0.032 & 1.210 & & \\
\hline \multicolumn{9}{|c|}{ Toxin byoshintetic process } \\
\hline SS1G_05105 & Putative uncharacterized protein & Sclerotinia sclerotiorum & 0.000 & -4.490 & 0.001 & -2.288 & 0.000 & -2.203 \\
\hline $\operatorname{stcC}$ & $\begin{array}{l}\text { Putative sterigmatocystin biosynthesis } \\
\text { peroxidase }\end{array}$ & Emericella nidulans & 0.004 & -4.080 & & & & \\
\hline tcmN & $\begin{array}{l}\text { Multifunctional cyclase-dehydratase-3-O- } \\
\text { methyl transferase }\end{array}$ & Streptomyces glaucescens & & & 0.000 & -3.530 & 0.000 & 2.798 \\
\hline & synthetase & & & & & & & \\
\hline
\end{tabular}




\section{Figures}
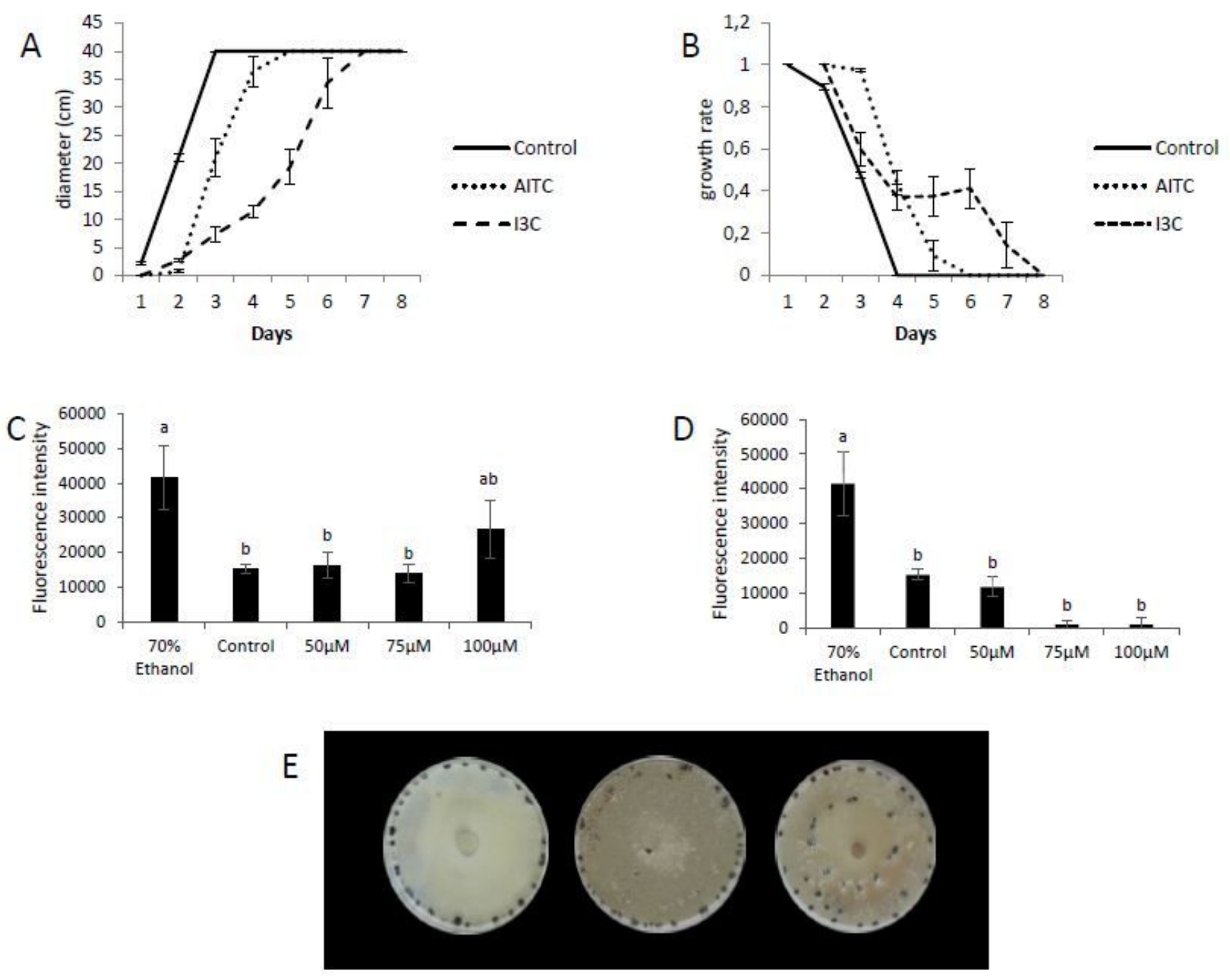

\section{Figure 1}

Radial growth A) and growth rate B) of Sclerotinia sclerotiorum in PDA medium with $100 \mu \mathrm{M}$ of allylisothiocyanate (AITC) or indol-3-carbinol (I3C) measured during eight consecutive days. Cell permeability assay of Sclerotinia sclerotiorum treated with AITC C) and I3C D) 40 minutes after the addition of SYTOX®-Green. Letters in the top of the bars indicate separation of means by least significant differences at $p \leq 0.05$. Production of sclerotia of Sclerotinia sclerotiorum grown in PDA medium under three different treatments (from left to the right): Control, AITC and I3C. 


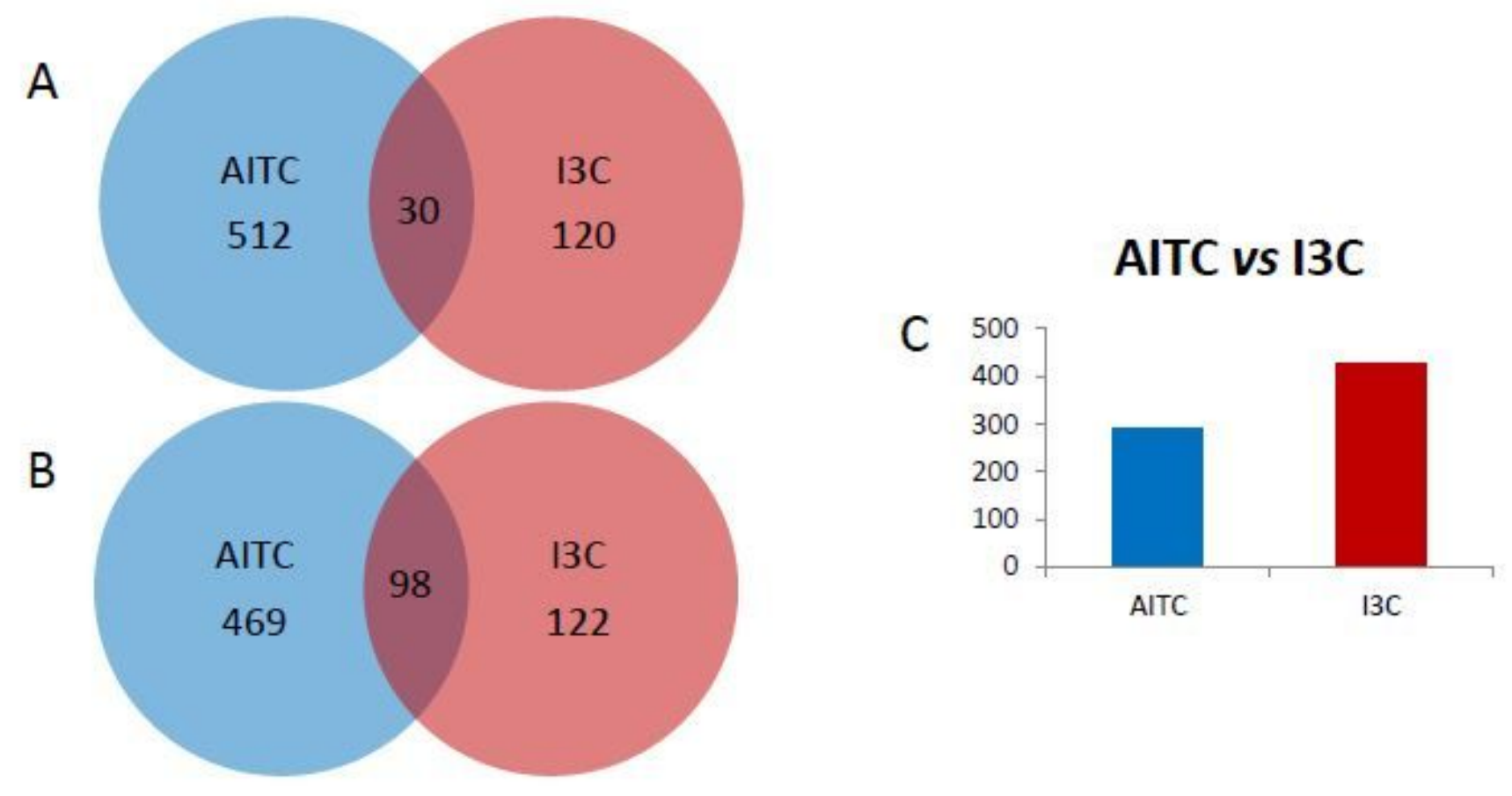

Figure 2

Venn graph showing overlapping A) up-differentially expressed genes (DEGs) and B) down-DEGs between the comparisons AITC vs Control and I3C vs Control. C) Number of DEGs up-regulated by AITC or I3C in the comparison AITC vs I3C. Identification of DEGs was performed based on a false discovery rate (FDR) $\leq 0.05$ and $-1 \leq \log 2$ fold change $(F C) \geq 1 \log 2$. 


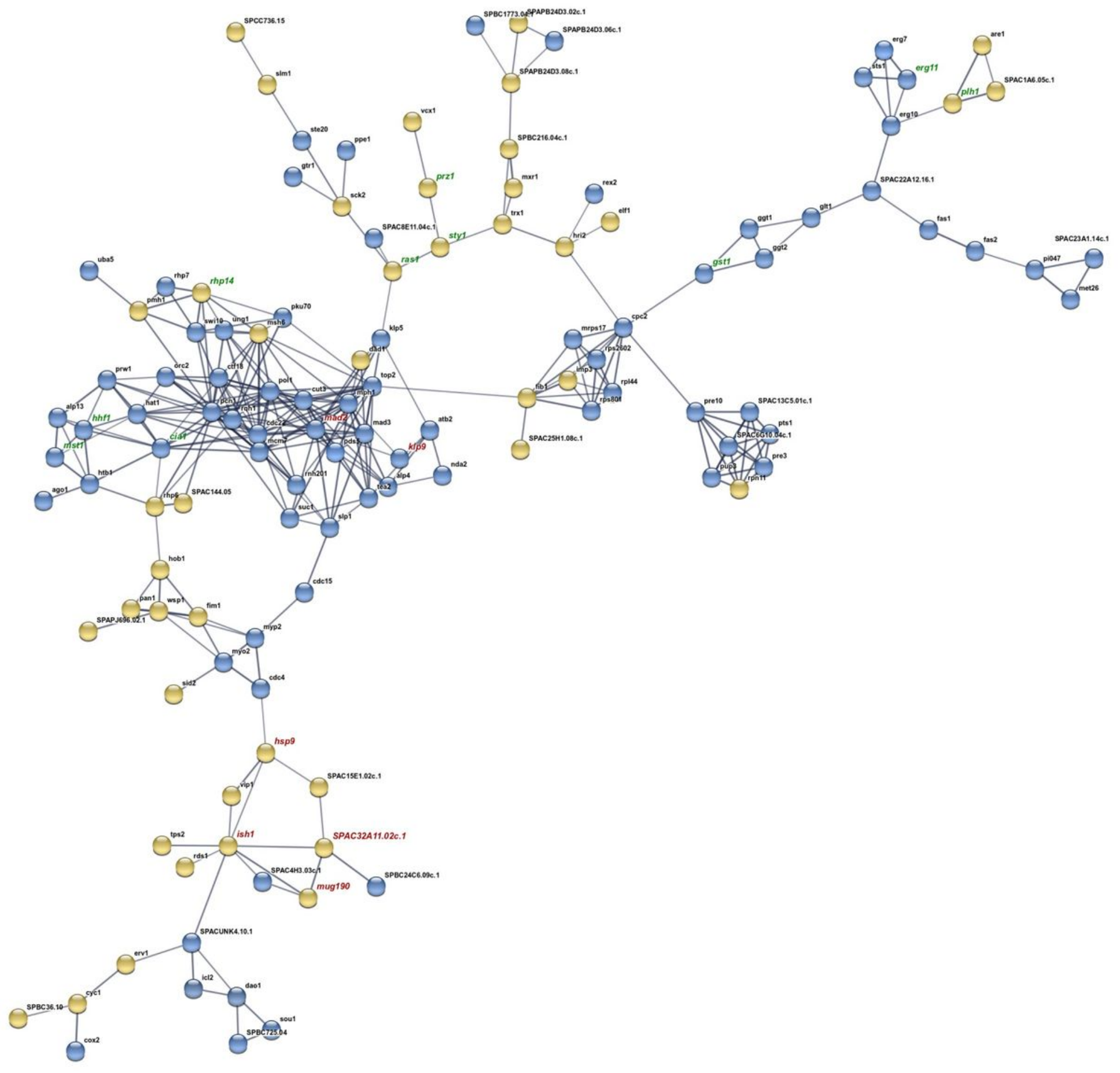

Figure 3

Protein association network built with all DEGs of the comparison AITC vs Control. Line thickness indicates the strength of data support, which is based on experimental results and co-expression analysis in Schizosaccharomyces pombe. Blue and yellow nodes indicate up or down-regulation of DEGs, respectively. Name of DEGs cited in the text are written in green. The name of DEGS shared with the comparison I3C vs Control are shown in red. Description of DEGs is given in Supplementary Table S2. 


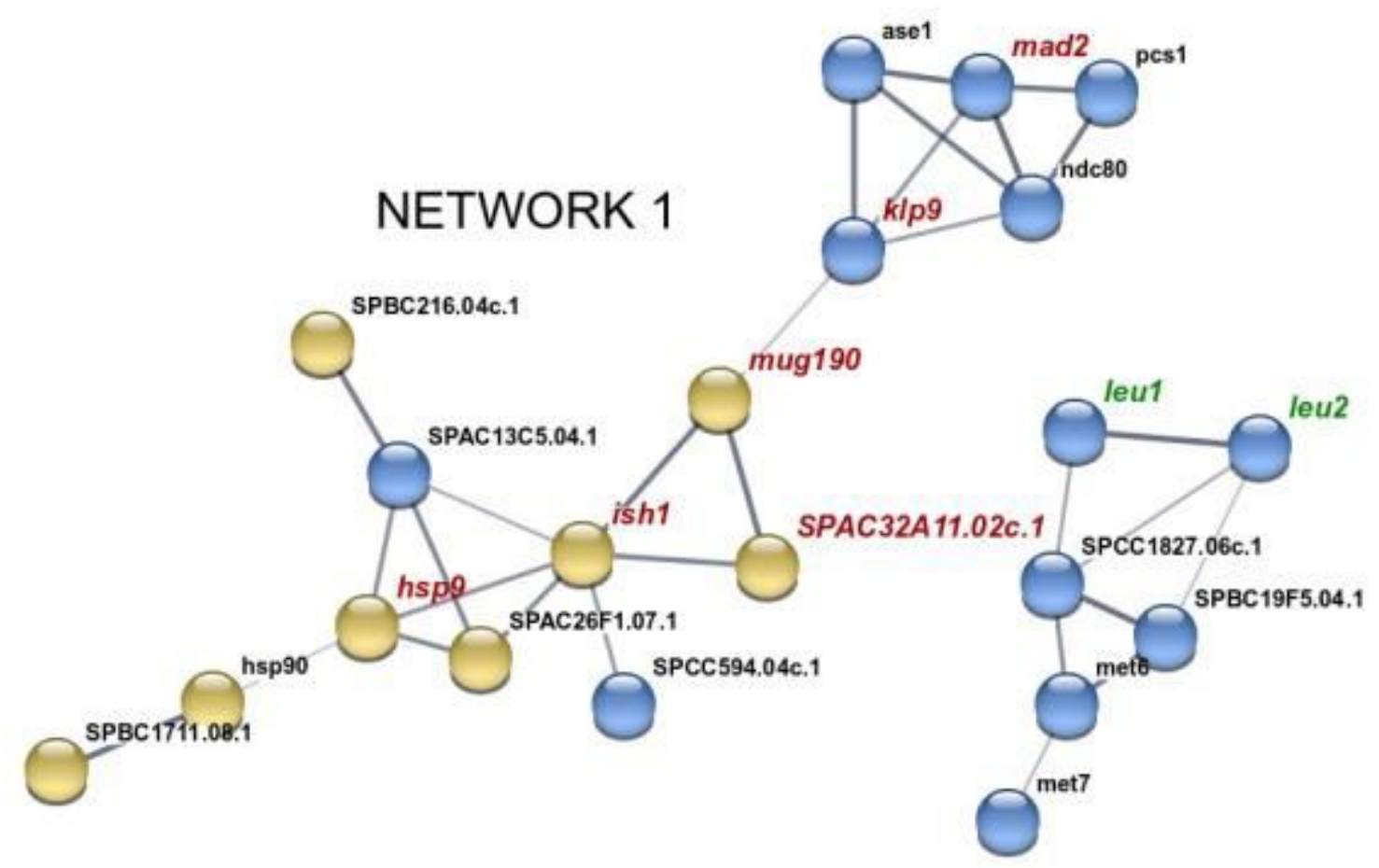

NETWORK 2

\section{Figure 4}

Protein association network built with all DEGs of the comparison I3C vs Control. Line thickness indicates the strength of data support, which is based on experimental results and co-expression analysis in Schizosaccharomyces pombe. Blue and yellow nodes indicate up or down-regulation of DEGs, respectively. Name of DEGs cited in the text are written in green. The name of DEGS shared with the comparison I3C vs Control are shown in red. Description of DEGs is included Supplementary Table S2. 


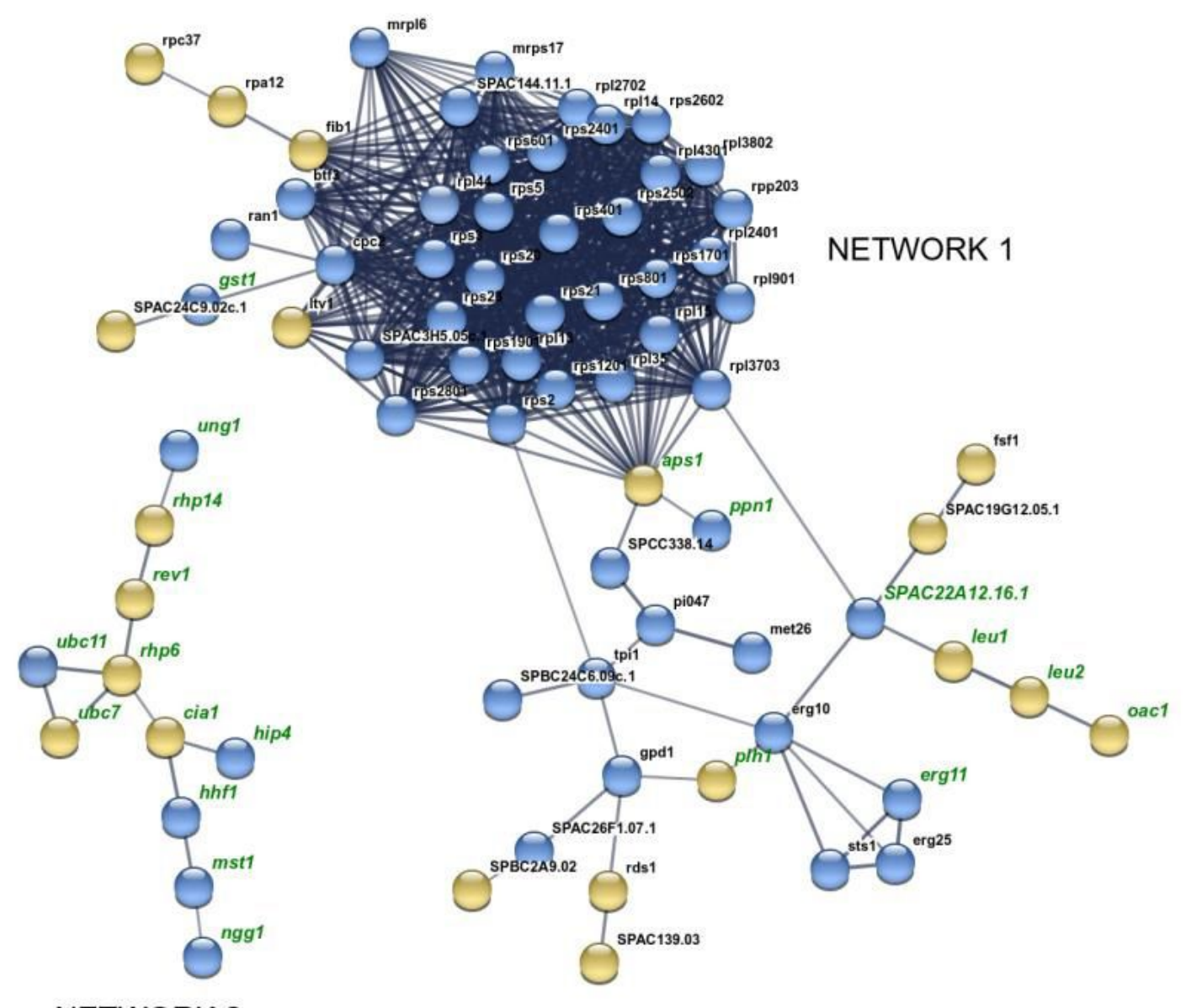

NETWORK 2

\section{Figure 5}

Protein association network built with all DEGs of the comparison AITC vs I3C. Line thickness indicates the strength of data support, which is based on experimental results and co-expression analysis in Schizosaccharomyces pombe. Blue nodes indicate up-regulated DEGs by AITC and yellow nodes indicate up-regulated DEGs by I3C, respectively. Description of DEGs is included in Supplementary Table S2. 


\section{Supplementary Files}

This is a list of supplementary files associated with this preprint. Click to download.

- SupplementaryTableS1.pdf

- SupplementaryTables2.pdf 